\title{
RELACIÓN DE LA EDAD CRONOLÓGICA CON LA MADURACIÓN ÓSEA CERVICAL MEDIANTE EL MÉTODO DE BACCETTI
}

\author{
RELATIONSHIP OF THE CHRONOLOGICAL AGE WITH CERVICAL BONE MATURATION \\ THROUGH THE BACCETTI METHOD \\ Juan Carlos Julca Lévano ${ }^{1}$ \\ jjulca@usat.edu.pe \\ ORCID: 0000-0001-5717-5475
}

\section{RESUMEN}

Introducción: Es importante valorar el momento ideal en el que se producen los cambios tanto biológicos como estructurales en el crecimiento y desarrollo de los maxilares, para identificar en qué momento se inicia del crecimiento puberal en los pacientes jóvenes y corregir las alteraciones tanto dentarias como esqueléticas que se puedan presentar. Objetivo: Evaluar los estadios de maduración de las vértebras cervicales mediante el análisis propuesto por Baccetti y su relación con la edad cronológica y el sexo, en radiografías laterales de individuos de 8 a 14 años. Metodología: Estudio transversal, retrospectivo y descriptivo. Se evaluaron 280 radiografías laterales (140 de varones y 140 de mujeres). Un evaluador calibrado evaluó los estadios de maduración esquelética e identificó 6 de ellas. Se evaluó el pico de crecimiento de los individuos desde la segunda a la cuarta vértebra cervical en las radiografías y luego se relacionó con la edad y el sexo de los individuos. Se utilizaron las pruebas de asociación de
Chi cuadrado $p<0,05$. Resultados: En las edades de 11 y 12 años se observó que las mujeres tenían mayor grado de madurez que los varones; sin embargo, para apreciar mejor los resultados, se reagruparon según los estadios: 1 con 2 , 3 con 4 y 5 con 6 , y se encontraron diferencias estadísticamente significativas entre ellos ( $p<0,001)$. Conclusiones: Existe asociación en el diagnóstico del pico de crecimiento y la maduración ósea de los pacientes según su edad cronológica, con una clara diferencia entre varones y mujeres, lo que evidencia que el método de Baccetti se pueda emplear de manera confiable en la población estudiada.

Palabras clave: edad, vértebras cervicales, maduración, individuo

\section{ABSTRACT}

Introduction: In Orthodontics, it is important to assess the ideal moment where changes occur, both biological and structural in relation to the growth and development of the jaws, thus identifying, at

Citar como: Julca Lévano JC. Relación de la edad cronológica con la maduración ósea cervical mediante el método de Baccetti. Rev Cient Odontol. (Lima). 2019; 7 (2): 42-51. 
what time, the onset of pubertal growth in young patients and substantiate their dental correction as skeletal, achieving a positive result through its planning and application, correcting its discrepancies or alterations that may occur. Objective: To evaluate the stages of maturation of the cervical vertebrae through the analysis proposed by Baccetti and its relationship with chronological age according to age and sex, on lateral head X-rays of individuals aged 8 to 14 years. Methodology: This was a crosssectional, retrospective, descriptive study. 280 lateral head X-rays were evaluated (140 men and 140 women). A trained and calibrated evaluator evaluated the stages of skeletal maturation with the method proposed by Baccetti, identifying 6 stages. The peak of growth of the individuals was evaluated through the second to the fourth cervical vertebra on radiographs and then related to the chronological age and sex of the individuals. Chi square association tests were used $p<$ 0.05. Results: In the ages of 11 and 12, it is observed that women have a greater degree of maturity than men, however, in order to better appreciate the results, they were regrouped according to the stages of maturity of cervical vertebrae 1 with 2, 3 with 4 and 5 with 6 and statistically significant differences were found between the stages of maturation of the cervical vertebrae according to age and sex $(p<0.001)$. Conclusions: There is an association in the diagnosis of peak growth and bone maturation of patients according to their chronological age, producing a clear difference between men and women, which shows that the Baccetti method can be used reliably in the population studied.

Keywords: age, cervical vertebrae, maturation, individual

\section{INTRODUCCIÓN}

En ortodoncia y ortopedia maxilar, es muy importante valorar el momento en el que se producen los cambios, tanto biológicos como estructurales, con relación al crecimiento y desarrollo de los maxilares, para identificar el inicio del crecimiento puberal y así fundamentar el tratamiento y corrección de las diferentes alteraciones tanto dentarias como esqueléticas $\left(^{1-4}\right)$, a fin de obtener un resultado positivo en el momento de la planificación y permitir que los diversos tipos de dispositivos funcionales corrijan las discrepancias o alteraciones que se puedan presentar $\left({ }^{5,6}\right)$. Para obtener la efectividad o una respuesta positiva en cuanto a la aplicación adecuada de los aparatos funcionales, es importante conocer y evaluar en gran medida la maduración esquelética y su potencial de crecimiento de forma individual en cada individuo, para así contar con información importante y anticiparnos a los resultados que nos proponemos en el tratamiento $\left({ }^{7-9}\right)$. Se debe de tener en cuenta que el porcentaje del crecimiento tanto de las estructuras esqueléticas como de las dentarias no es constante en el desarrollo $\left({ }^{9,10}\right)$.

En cuanto a la edad cronológica, es decir, desde el nacimiento hasta la edad actual del paciente, no le permite al clínico clasificar los periodos en los que se produce el aceleramiento, disminución o detención del crecimiento. En cuanto al desarrollo y madurez de las personas, recordemos que este es único, individual y propio, para lo cual tenemos diversos indicadores biológicos: altura corporal, maduración esquelética de mano y muñeca, formación y erupción dental, menarquía, cambios en la agudeza de voz y maduración cérvico-vertebral $\left({ }^{11-17}\right)$. Por lo tanto, el concepto de maduración se relaciona con la edad biológica de cada individuo, en la que ocurren cambios físicos y biológicos tanto a nivel celular como de 
órganos, tejidos y sistemas relacionados con la edad cronológica; pero las diferencias entre ellas y entre cada individuo pueden ser muy marcadas $\left({ }^{18-20}\right)$.

La valoración de la maduración de las vértebras cervicales ha demostrado ser un método que presenta una alta y recíproca relación con el análisis de maduración ósea mano-muñeca (21-24). Por tanto, en este estudio se utilizan las radiografías laterales de cabeza que se solicitan en ortodoncia y se evita así el uso de radiografías adicionales y, lo más importante, la exposición adicional del paciente a la radiación ${ }^{25-29}$.

Existen diversas investigaciones, de diversos lugares, que han comprobado la validez, importancia y aplicación de este análisis. Los estudios en comunidades latinas son muy pocos en cuanto a su correlación. Las investigaciones demuestran que en casi el $95 \%$ de los sujetos de América del Norte coincide el crecimiento puberal tanto con el desarrollo mandibular como con la altura corporal. La posibilidad de clasificar las etapas de maduración de las vertebras cervicales es alta, con casi un $98 \%$ por parte de examinadores capacitados. Esto demuestra que se trata de un método confiable en comparación con el análisis mano-muñeca $\left({ }^{30,31}\right)$.

La importancia del estudio radica en identificar el momento ideal en el que se pueden realizar cambios en los múltiples tipos de maloclusiones y aplicar adecuadamente una aparatatología funcional para modificar estas alteraciones, en cuanto a su crecimiento y desarrollo en los maxilares. Por tanto, el propósito de este estudio fue evaluar, mediante el análisis propuesto por Baccetti, la importancia de su aplicación y su relación con los estadios de maduración de las vértebras cervicales, para determinar así su relación con la edad cronológica según el sexo de los evaluados, mediante el uso de radiografías laterales de cabeza de individuos entre 8 y 14 años.

\section{MATERIALES Y MÉTODOS}

Este estudio retrospectivo, descriptivo, observacional y de corte transversal fue aprobado por el Comité Institucional de Ética de la Universidad Científica del Sur mediante el código de registro 091-2019POS8.

La unidad de análisis estuvo conformada por todas las radiografías laterales de cabeza que se encuentran en el archivo de pacientes que asistieron a un centro radiológico en la ciudad de Chiclayo. El tamaño muestral fue de 280 radiografías laterales, con distribución de género y edades de 8 a 14 años, considerando que las tomas radiográficas fueron de un centro radiológico y evaluadas según el análisis propuesto por Baccetti. En cuanto a las variables que se utilizaron, fueron edad, sexo y análisis de las vértebras cervicales. Los criterios de inclusión que se utilizaron fueron radiografías nítidas, con buen contraste en la zona de las vértebras cervicales, de individuos con apellidos hispanoamericanos que acudieron a un centro radiológico en la ciudad de Chiclayo y que no presenten alteraciones de accidentes, lesiones o traumas en cuello. Cabe resaltar que el presente estudio se realizó sin registrar los nombres y apellidos, o algún otro dato que pudiera ser usado en la identificación de los pacientes, para salvaguardar la estricta confidencialidad de la muestra, por lo que no existió ninguna vulneración ética al respecto.

El procedimiento aplicado se basó en la evaluación del estadio óseo de maduración cérvico-vertebral $\left({ }^{11}\right)$.

Este análisis, de tipo visual, se estableció en dos puntos principales: 
1. Concavidad de las vértebras $\mathrm{C} 2, \mathrm{C} 3$ y $\mathrm{C} 4$, a nivel del borde o límite inferior de los cuerpos cérvico-vertebrales

2. Forma de los cuerpos vertebrales de la C3 y C4 (rectangular, horizontal, trapezoidal, rectangular vertical $\mathrm{o}$ cuadrado

En cuanto a los estadios óseos de maduración cervical-vertebral, según Baccetti $\left({ }^{11}\right)$, son los siguientes:

Estadio CS1. La vértebra C2 presenta un borde o límite inferior totalmente plano. Un $7 \%$ puede presentar anatómicamente una concavidad. A nivel de las vértebras $\mathrm{C} 3$ y $\mathrm{C} 4$, se observa una anatomía trapezoidal. A nivel mandibular, el pico de crecimiento en este estadio se presenta 24 meses luego de esta etapa.

Estadio CS2. La vértebra C2 presenta un borde o límite inferior con una concavidad en un $80 \%$ de los casos. Las vértebras $\mathrm{C} 3$ y $\mathrm{C} 4$ mantienen anatómicamente la forma trapezoidal. A nivel mandibular, el pico de crecimiento se inicia luego de 12 meses de esta etapa.

Estadio CS3. Las vértebras C2 y C3 presentan un borde o límite inferior con una concavidad. Las vértebras $\mathrm{C} 3$ y C4 pueden tener una forma rectangular horizontal o trapezoidal. A nivel mandibular, el pico de crecimiento se inicia en esta etapa.

Estadio CS4. Las vértebras C2, C3 y $\mathrm{C} 4$, en su totalidad, muestran concavidades en sus bordes. Las vértebras $\mathrm{C} 3$ y C4 presentan una anatomía de forma rectangular horizontal. A nivel mandibular, el pico de crecimiento termina en esta etapa o 12 meses antes de ella.

Estadio CS5. Las vértebras C2, C3 y C4 suelen presentar una concavidad en sus bordes o limites inferiores. En el caso de las vértebras C3 y C4, una de ellas presenta forma cuadrada y, si no es así, el cuerpo de la otra mantiene su forma rectangular horizontal. A nivel mandibular, el pico de crecimiento ha terminado 12 meses antes de esta etapa.

Estadio CS6. Las vértebras C2, C3 y C4 presentan una concavidad muy marcada. En cuanto a los cuerpos vertebrales de la $\mathrm{C} 3$ y la $\mathrm{C} 4$, al menos una de ellas tiene una anatomía rectangular vertical. A nivel mandibular, el pico de crecimiento ha terminado 24 meses antes de esta etapa (figura 1).

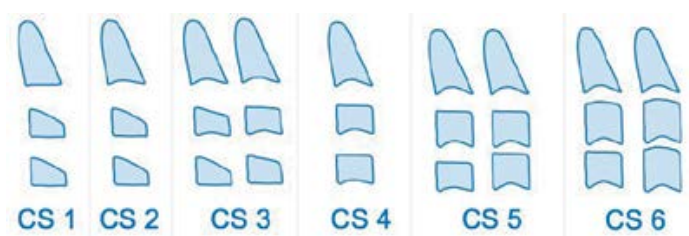

Modelo de evaluación de las vértebras cervicales según el análisis propuesto por Baccetti.

\section{FIgURA 1. MÉtodo de MAdURACIÓN DE LAS VÉRTEBRAS CERVICALES (CVM)}

Las imágenes radiográficas fueron analizadas en una laptop de la marca Dell, Core i7 de 8.a generación, visualizadas en una pantalla de 19 pulgadas de tipo panorámico, con una resolución de 1920 x 1080 píxeles en un ambiente con luz tenue. El contraste y brillo de las imágenes fueron ajustados usando la herramienta procesadora de imágenes del software para asegurar una visualización óptima. Las muestras se evaluaron en dos momentos distintos, con un intervalo de 10 días entre una y otra, y luego fueron comparadas con las del Gold Estándar y analizadas utilizando el programa estadístico SPSS versión 25 (SPSS Inc., Chicago ILL). Se halló que el kappa de Cohen alcanza el valor de 0,88 , lo que demuestra un alto nivel del concordancia.

El análisis estadístico para establecer las diferencias entre los estadios de acuerdo con el sexo de las personas empleó la 
prueba de Chi cuadrado de independencia de criterios, con un nivel de significancia de $p<0,05$.

\section{RESULTADOS}

El total de la muestra fueron 280 radiografias laterales, divididas en 140 (50\%) de varones y $140(50 \%)$ de mujeres, y por edades (20 placas por cada edad y sexo), entre 8 y 14 años.

Para contrastar las hipótesis se trató de establecer las diferencias entre los niveles de diagnóstico de acuerdo con el sexo en cada grupo de edad.

La tabla 1 presenta resultados en los cuales se observa que hay un cambio en la madurez cervical diferencial entre hombres y mujeres a los 11 y 12 años.
La tabla 2 presenta resultados en los que las mujeres maduran antes que los hombres. Antes y después de estas edades, el diagnóstico de madurez es similar en ambos sexos. Al realizar la comparación de los estadios de madurez con respecto a las edades de los pacientes, se aprecian diferencias.

La tabla 3 presenta resultados en los que, conforme se incrementa la edad, los estadios de mayor madurez van predominando. Sin embargo, para apreciar mejor estos resultados se reagruparon los estadios de madurez de las vértebras cervicales de la siguiente forma: $\mathrm{G} 1$, estadios 1 con 2; G2, estadios 3 con 4 ; y G3, estadios 5 con 6 . Se observa mejor el incremento del número de pacientes en los estadios mayores, conforme aumenta su edad.

TABLA 1. RELACIÓN ENTRE EL SEXO Y EL TIPO dE DIAGNóstiCo EN PACIENTES DE 8 A 14 AÑOS

\begin{tabular}{|c|c|c|c|c|c|c|c|c|}
\hline \multirow{2}{*}{ Edad (años) } & \multirow{2}{*}{ Sexo } & \multicolumn{6}{|c|}{ DIAGNÓSTICO SEGÚN EDAD Y SEXO } & \multirow{2}{*}{ p-valor } \\
\hline & & 1 & 2 & 3 & 4 & 5 & 6 & \\
\hline \multirow[t]{2}{*}{8} & hombre & 6 & 9 & 4 & 1 & 0 & 0 & 0,783 \\
\hline & mujer & 7 & 9 & 4 & 0 & 0 & 0 & \\
\hline \multirow[t]{2}{*}{9} & hombre & 4 & 6 & 5 & 5 & 0 & 0 & 0,067 \\
\hline & mujer & 0 & 4 & 3 & 12 & 1 & 0 & \\
\hline \multirow[t]{2}{*}{10} & hombre & 0 & 3 & 2 & 13 & 2 & 0 & 0,124 \\
\hline & mujer & 4 & 2 & 3 & 6 & 4 & 1 & \\
\hline \multirow[t]{2}{*}{11} & hombre & 6 & 6 & 6 & 2 & 0 & 0 & $<0,001^{*}$ \\
\hline & mujer & 0 & 1 & 3 & 7 & 7 & 2 & \\
\hline \multirow[t]{2}{*}{12} & hombre & 0 & 4 & 4 & 8 & 0 & 4 & $0,009^{*}$ \\
\hline & mujer & 3 & 1 & 1 & 3 & 6 & 6 & \\
\hline \multirow[t]{2}{*}{13} & hombre & 5 & 2 & 4 & 2 & 3 & 4 & 0,108 \\
\hline & mujer & 3 & 1 & 1 & 8 & 6 & 1 & \\
\hline \multirow[t]{2}{*}{14} & hombre & 2 & 2 & 3 & 7 & 5 & 1 & 0,510 \\
\hline & mujer & 1 & 0 & 1 & 9 & 8 & 1 & \\
\hline
\end{tabular}


Tabla 2. Relación entre las edades de 8 a 14 años y el tipo de diagnóstico

\begin{tabular}{|l|c|c|c|c|c|c|c|}
\hline \multicolumn{1}{|c|}{ Edad } & \multicolumn{9}{|c|}{ Diagnóstico del estadio vértebro-cervical } & \multirow{2}{*}{ p-valor } \\
\hline 8 años & 1 & 2 & 3 & 4 & 5 & 6 & \\
\hline 9 años & 13 & 18 & 8 & 0 & 0 & 0 & \\
\hline 10 años & 4 & 10 & 8 & 0 & 1 & 0 & \\
\hline 11 años & 4 & 5 & 5 & 1 & 6 & 1 & \\
\hline 12 años & 3 & 7 & 9 & 2 & 7 & 2 & \multirow{2}{*}{$00,001^{*}$} \\
\hline 13 años & 8 & 3 & 5 & 10 & 6 & 10 & \\
\hline 14 años & 3 & 2 & 4 & 2 & 13 & 2 & \\
\hline TOTAL & 41 & 50 & 44 & 20 & 42 & 20 & \\
\hline *significativo & & & & & & & \\
Prueba de Chi cuadrado & & & & & &
\end{tabular}

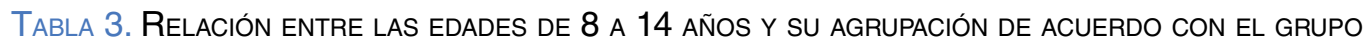
DIAGNÓSTICO

\begin{tabular}{|c|c|c|c|c|}
\hline \multirow{2}{*}{ Edad } & \multicolumn{3}{|c|}{ Dx1 agrupado } & \multirow{2}{*}{$\mathrm{p}$-valor } \\
\hline & G1 & $\mathrm{G} 2$ & G3 & \\
\hline 8 años & 31 & 9 & 0 & \multirow{8}{*}{$<0,001^{*}$} \\
\hline 9 años & 14 & 25 & 1 & \\
\hline 10 años & 9 & 24 & 7 & \\
\hline 11 años & 13 & 18 & 9 & \\
\hline 12 años & 8 & 16 & 16 & \\
\hline 13 años & 11 & 15 & 14 & \\
\hline 14 años & 5 & 20 & 15 & \\
\hline Total & 91 & 127 & 62 & \\
\hline
\end{tabular}

\section{DISCUSIÓN}

Durante mucho tiempo se ha utilizado la radiografía de mano-muñeca para determinar el nivel de maduración de un paciente niño. Este método ha demostrado ser eficiente y evita la radiación adicional ${ }^{25}$ ). La radiografía lateral de cabeza demostró ser un método tan válido como el análisis de mano-muñeca, por su factibilidad y la reducción de la exposición a la radiación ionizante de los pacientes en crecimiento. La modificación de las vértebras cervicales en cuanto a su dimensión y forma ha demostrado, en los últimos años, confiabilidad en la evaluación de la tasa de maduración esquelética de un individuo. Sus implicancias en el área de la ortodoncia han sido muy importantes, por su eficacia en el análisis de las vértebras cervicales para evaluar la edad esquelética $\left.{ }^{(32}\right)$.

La aplicación del método de maduración de las vértebras cervicales puede ser útil para detectar el momento ideal y óptimo para iniciar el tratamiento de las deficiencias esqueléticas por medio de la ortodoncia y la ortopedia funcional. En cuanto a las variaciones anatómicas de 
las vértebras cervicales de pacientes en proceso de crecimiento, ha demostrado ser un indicador biológico individual más popular dado que se realiza en una radiografía lateral o de perfil para evaluar las etapas o estadios, por lo que es usado como ayuda para el diagnóstico. Los resultados del método describen que se produce el pico de crecimiento mandibular en los estadios III y IV, lo que indica el momento ideal para el tratamiento ortopédico.

La determinación de la concordancia entre los estadios de calcificación y los estadios de maduración esquelética, también presentan resultados variados en estudios previos $\left({ }^{16,16,31,33}\right)$. En cuanto a las vértebras cervicales y su correlación con las C2, C3 y C4 y la edad cronológica, esta va en aumento proporcionalmente y se encuentra una gran prevalencia del estadio o etapa II, que es el ideal para el inicio de la terapéutica ortodóntica y ortopédica, con lo que demuestra ser un método muy útil y confiable para determinar el crecimiento facial en su etapa puberal $\left({ }^{2}\right)$. La evaluación de la maduración esquelética mediante el análisis de las vértebras cervicales proporciona datos importantes para la planificación, pero otras investigaciones sugieren asociar otros indicadores de maduración esquelética - a pesar de haberse demostrado estadísticamente su confiabilidad y validez en pacientes adolescentes-, como la maduración sexual, el crecimiento corporal, etc. $\left({ }^{26}\right)$. Se logró determinar la variación de la edad cronológica con relación al estadio de maduración ósea cervical según el sexo en relación con la edad cronológica, que muestra mayor evidencia en el estadio C3 $\left({ }^{9}\right)$.

Los resultados obtenidos demostraron que hay relación entre las edades, según edad y sexo, y los estadios de maduración ósea mediante el análisis propuesto por Baccetti, y se encontraron diferencias estadísticamente significativas en las niñas en cuanto a su maduración, que es más acelerada que en los niños. Asimismo, se demostró que entre las edades de 11 y 12 años había una relación entre el sexo y su estadio, debido a que las frecuencias entre ambos son diferentes. Como se pudo apreciar, los hombres tienen sus mayores frecuencias en las etapas 1, 2, 3 y 4, mientras que las mujeres tienen mayores frecuencias en 4, 5 y 6 . En general, se observó que, en las edades de 8 y 9 años, los estadios 1, 2 y 3 son los que presentan mayor frecuencia; en las edades de $10 \mathrm{y}$ 11 años, se presentan frecuencias similares, mientras que en las edades de 12, 13 y 14 años, los estadios 4,5 y 6 presentan mayores frecuencias. Se pudo apreciar más claramente que a partir de los 12 años presentan estadios mayores; en cambio, antes de esa edad predominan los estadios menores, por lo cual se tuvo que agrupar 1 y 2 en $\mathrm{G} 1,3$ y 4 en G2, y 5 y 6 en G3, para luego establecer la relación con respecto a la edad.

Por lo tanto, se debe considerar este análisis como una herramienta de ayuda al diagnóstico, porque nos permite valorar los estadios de maduración de las vértebras cervicales, análisis que se puede ampliar para futuras investigaciones mediante la utilización de otros medios de ayuda al diagnóstico, como la tomografía cone beam, que permitiría apreciar otra dimensión (3D), su anatomía y así analizar y comprobar su relación.

\section{CONCLUSIONES}

El uso de la radiografía lateral de cabeza demostró ser un análisis confiable para la evaluación de las vértebras cervicales utilizando el análisis propuesto por Baccetti, lo que permite proyectar o estimar el pico de crecimiento y la maduración ósea, y ayuda al diagnóstico y la planificación de un tratamiento. En 
conclusión, este análisis nos confirma que puede determinar la edad cronológica y su relación con la maduración ósea según edad y sexo, por lo que es una herramienta de gran ayuda para el diagnóstico, así como para el profesional, al obtener una relación positiva entre ambas entidades mediante la maduración de las vértebras cervicales que va acorde con la edad cronológica. Además, muestra diferencias estadísticamente significativas entre mujeres de 8 a 14 años y hombres, pues las primeras tienen un estadio de maduración ósea más avanzado, lo que demuestra la diferencia en función del sexo $(p=<0,001)$.

Contribución del autor: Juan Carlos Julca Lévano ha participado en la concepción del artículo, la recolección de información, la redacción y la aprobación de la versión final.

Fuente de financiamiento: Autofinanciado

Conflicto de interés: Ninguno

\section{REFERENCIAS BIBLIOGRÁFICAS}

1. Li Chen L, Min Xu T, Hui Jiang J, Zhong Zhang X, Xiang Lin J. Quantitative cervical vertebral assessment in adolescents with normal occlusion: A mixed longitudinal study. Am Assoc of Orthod. 2009; 134 (6): 720.e1-e7.

2. Muglia V, Lederman $H$, Moscatiello R, Junior K, Moscatiello V. Maturação das vértebras cervicais e sua correlação com a idade óssea da mão e punho como indicadores no tratamento ortodóntico. Dental Press Orthod Orthop Facial. 2008; 13 (4): $92-100$.

3. McNamara J. Long-term adaptations to changes in the transverse dimension in children and adolescents: An overview. Am J Orthod Dentofacial Orthop. 2006; 129: S71-40889-5406.

4. González MC, Martínez CM, Mora I, Bautista G, Palmet S. Estado de maduración ósea de las vértebras cervicales en una población colombiana con y sin labio y paladar surado. Univ Odontol. 2014; 33 (70): 41-50. 
5. Ortiz M, Godoy S, Fuenmayor D, Farias M, Quirós O, Rondón S, Lerner H. Método de maduración ósea de las vértebras cervicales, en pacientes del Diplomado de Ortodoncia Interceptiva. UGAMA, 2006.

6. Baccetti T, Franchi L, McNamara Jr. An improved version of the cervical vertebral maturation (CVM) method for the assessment of mandibular growth. Angle Orthod. 2002; 72 (4): 316-23.

7. Safer A, Homel P, Chung D. Lateral comparisons using Fishman's skeletal maturation assessment. Angle Orthod. 2015; 85 (3) :408-12.

8. Ávila M. Determinación del pico máximo de crecimiento puberal mandibular mediante la maduración ósea cérvico vertebral y la edad cronológica en pacientes adolescentes que acuden a la clínica dental docente UPC marzo 2011-2013. [Tesis de licenciatura]. Lima: Universidad Peruana de Ciencias Aplicadas; 2015.

9. Bedoya A, Osorio J, Tamayo J. Chronological age and cervical vertebral maturation among children and adolescents. Rev Cubana de Estom. 2016; 51 (1): 43-53.

10. McNamara Jr. J, Franchi L. The cervical vertebral maturation method: A user's guide. Angle Orthod. 2018; 88: 133-43.

11. Bedoya A, Osorio J, Tamayo J. Chronological age and cervical vertebral maturation among children and adolescents. Rev Cubana Estom. 2016; 51 (1): 43-53.

12. Gladia M, Rigobert L. Assessment of bone maturation in cervical vertebrae in Orthodontics patients. Rev. Cubana Estomatol. 2010; 47 (3).

13. Kalinowska I, Raczka A, Kalinowski P. Relationship between dental age according to Demirjian and cervical vertebrae maturity in polish children. Eur J Orthod. 2011; 33: 75-83.

14. Ball G, Woodside D, Tompson B, Hunter W, Posluns J. Relationship between cervical vertebral maturation and mandibular growth. Am J Orthod Dentofacial Orthop. 2011; 139: e455-e461.

15. Franchi L, Baccetti T, De Toffol L, Polimeni A, Cozzad P. Phases of the dentition for the assessment of skeletal maturity: a diagnostic performance study. Am J Orthod Dentofacial Orthop. 2008; 133 (3): 395-400.

16. Baccetti T, Franchi L, McNamara, Jr. J. The Cervical Vertebral Maturation (CVM) method for the assessment of optimal treatment timing in dentofacial orthopedics. Semin Orthod 2005; 11: 119-29.

17. Ceglia A. Indicadores de maduración de la edad ósea dental y morfológica. Rev Latin de Ortod y Odp. 2005.

18. Santiago R, De Miranda L, Farinazzo R, Reis M, Bolognese A, Cople Lucianne. Cervical vertebral maduration as a biologic indicator of skeletal maturiry a systematic review. Angle Orthod. 2012; 82: 1123-31.

19. Salazar R, Moya T. Bone maturation evaluation through Baccetti cervical vertebrae study method. Dom. Cien. 2017; 3 (1): 373-88. 
20. Plazas J, Martínez O, Herrera A. Maduración esquelética mediante análisis de Baccetti. Rev. Cient.Soc. Colomb.Ortod. 2015; 2 (2): 105-9.

21. Salazar R, Moya J. Bone maturation evaluation through Baccetti cervical vertebrae study method. Dom. Cien. 2017; 3 (1): 373-88.

22. Golaszewski A. Maduración esqueletal según el método de evaluación cervicovertebral. ¿Qué información nos proporciona el estudio de las vértebras cervicales? Rev Latam Ort y Odp. 2014.

23. Pinares J. Estimación de la edad cronológica en población chilena a través del estudio radiográfico del desarrollo del tercer molar. [Tesis de maestría]. Santiago de Chile: Universidad de Chile; 2015.

24. Morales E, Martínez C, Gonzales E, Canseco J, Cuairán V. Existing relationships between the size and shape of the cervical vertebrae wih the stages of carpal bone maturation. Rev Mex Ortodon. 2014; 2 (1): 24-31.

25. San Román P, Palma Juan, Oteo Ma, Nevado E. Skeletal maturation determined by cervical vertebrae development. Eur J Orthod. 2002; 24 (3): 303-11.

26. Xiao-Guang Z, Jiuxiang L, Jiu-Hui J, Qingzhu W, Sut Hong N. Validity and reliability of a method for assessment of cervical vertebral maturation. Angle Orthod. 2012; 82: 229-34.

27. Alvarado E, Gutiérrez J, Rivas R. Evaluatión of the cervical vertebral maturatión. (Baccetti method) in patients aged 8 to 15 years. Int. J. Odontostomat. 2016; 10 (1): 63-7.

28. Safer A, Homel P, Chung D. Lateral comparisons using Fishmans skeletal maturation assessment. Angle Orthod. 2015; 85 (3): 408-12.

29. Mulett J, Parra H. Ossification characteristics of the hand and the cervical vertebrae and correlation between the two techniques as indicators of somatic maturation in children from 8 to 17 years. Rev. Est. 2012; 20 (2):7-15.

30. Baccetti T. Entrevista. Dental Press J Orthod. 2011; 16 (4): 25-31.

31. Franchi L, Baccetti T, McNamara Jr. JA. Mandibular growth as related to cervical vertebral maturation and body height. Am J. Orthod Dentofacial Orthop 2000; 118 (3): 335-40.

32. Gandini P, Mancini M, Andreani F. A comparison of hand-wirst bone and cervical vertebral analysis in measuring skeletal maturation. Angle Orthod. 2006; 76 (6):984-9.

33. Rojas A, Torres A. Características de maduración esquelética y su relación con la edad cronológica y dental en niños de 5 a 16 años de la ciudad de Bucaramanga. [Tesis de grado]. Bucaramanga: Universidad Santo Tomás; 2016. 